\title{
Meridional circulation, turbulence and lithium in sub-giants originating from the hot side of the dip
}

\author{
Suzanne Talon \\ Départ. de Physique, Université de Montréal, and CERCA, Canada \\ Corinne Charbonnel \\ Laboratoire d'Astrophysique de Toulouse, France
}

\begin{abstract}
We present the impact of meridional circulation and shear turbulence on the evolution of the lithium abundance at the surface of evolved stars originating from the hot side of the Li Dip. We show that our fully consistent treatment of the same hydrodynamical processes which can account for $\mathrm{C}$ and $\mathrm{N}$ anomalies in B type stars (Talon et al. 1997) and for the shape of the hot side of the Li dip in open clusters (Talon \& Charbonnel 1998) also explains Li observations in stars with $\mathrm{T}_{\text {eff }}$ higher than $7000 \mathrm{~K}$ on the main sequence as well as in their evolved counterparts (see also Charbonnel \& Talon 1999).
\end{abstract}

\section{The problem of $\mathrm{Li}$ in subgiants}

We focus here on stars originating from the hot side of the Li dip (early-F and A types). Such stars are known to have $\mathrm{Li}$ abundances close to the cosmic value at the age of the Hyades. However, their subgiant counterparts show significant $\mathrm{Li}$ depletion much before dilution by the dredge-up takes place in standard models. These data reflect different degrees of depletion of $\mathrm{Li}$ inside the star while on the main sequence which do not show up at the stellar surface at the age of the Hyades.

\section{The models vs the observations}

We show how rotational mixing, based on wind driven meridional circulation and shear, modifies the $\mathrm{Li}$ abundance. We treat meridional circulation as a truly advective process (cf. Zahn 1992), whereas shear is treated via a turbulent viscosity (Talon \& Zahn 1997). We also consider the settling of those elements for which radiative forces are known to be small ( $\mathrm{He}, \mathrm{C}, \mathrm{N}, \mathrm{O}, \mathrm{Ne}$ and $\mathrm{Mg}$ ). We built models with masses ranging from 1.5 to $2.2 M_{\odot}$ with a solar metallicity. Initial velocities range from 50 to $150 \mathrm{~km} . \mathrm{s}^{-1}$. We consider two different cases. (1) Models conserving their global angular momentum (see also Charbonnel \& Talon 1999). Such models do preserve most of their surface lithium at the age of the Hyades while more depletion occurs in their interior (compared to standard models), resulting in extra surface depletion on the sub-giant branch. (2) Stars 


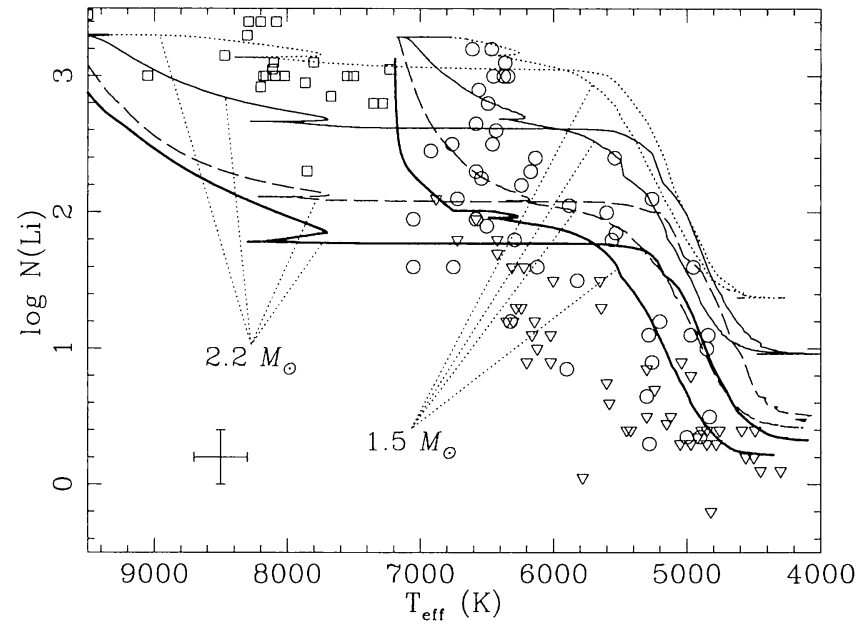

Figure 1. Evolution of surface lithium with effective temperature. The solid, dashed and dashed-dotted lines correspond respectively to the models with initial rotation velocities of 100,50 and $150 \mathrm{~km} \cdot \mathrm{sec}^{-1}$. Heavy lines are for models loosing a small amount of angular momentum on the main sequence. Dots and triangles : sub-giants observed by Lèbre et al. (1999) and Wallerstein et al. (1994). Squares : main sequence stars observed by Burkhart \& Coupry $(1998,2000)$.

loosing a very small amount of angular momentum during their main sequence lifetime, resulting into a very small slowdown compatible with the measurements of surface velocities at the age of the Hyades. In such a case, more depletion occurs, leading to earlier depletion of lithium on the sub-giant branch. Figure 1 shows the comparison of our theoretical predictions with observations in subgiants. Whereas the models which completely conserve their angular momentum predict fairly well the final amount of mixing, that mixing occurs somewhat too late. Including a small amount of braking (which agrees with the observations) takes care of that problem.

\section{References}

Burkhart C., Coupry M.F., 1998, A\&A 338, 1073

Burkhart .C., Coupry M.F., 2000, A\&A, accepted

Charbonnel C., Talon S., 1999, A\&A, 351, 635

Lèbre A., de Laverny P., De Medeiros J.R., Charbonnel C., da Silva L., 1999, A\&A 345, 936

Talon S., Charbonnel C., 1998, A\&A, 335, 959

Talon S., Zahn J.P., Maeder A., Meynet G., 1997, A\&A 322, 209

Talon S., Zahn J.P., 1997, A\&A 317, 749

Wallerstein G., Bohn-VItense E., Vanture A.D., Gonzalez G., 1994, AJ, 107, 221

Zahn J.-P., 1992, A\&A 265, 115 